\title{
The Association of Plasma Fluorescent Oxidation Products and Chronic Kidney Disease: A Case-Control Study
}

\author{
Casey M. Rebholz ${ }^{a}$ Tianying Wu ${ }^{d}$ L. Lee Hamm ${ }^{b, c}$ Robin Arora ${ }^{\mathrm{e}}$ \\ Islam E. Khan ${ }^{b}$ Yanxi Liu ${ }^{a}$ Chung-Shiuan Chen ${ }^{a}$ Katherine T. Mills ${ }^{a}$ \\ Stephanie Rogers $^{f}$ Myra A. Kleinpeter ${ }^{b}$ Eric E. Simon ${ }^{b}$ Jing Chen ${ }^{a-c}$ \\ ${ }^{a}$ Department of Epidemiology, Tulane University School of Public Health and Tropical Medicine, ${ }^{b}$ Department of \\ Medicine, and ${ }^{\mathrm{C} T u l a n e}$ Hypertension and Renal Center of Excellence, Tulane University School of Medicine, \\ New Orleans, La., d Department of Environmental Health, University of Cincinnati College of Medicine, \\ Cincinnati, Ohio, ${ }^{e}$ Kumar Nephrology and Hypertension, Huntington, W. Va., and ${ }^{\mathrm{f}}$ Department of Medicine, \\ University of California San Francisco, San Francisco, Calif., USA
}

\section{Key Words}

Albuminuria - Biological markers • Case-control study • Glomerular filtration rate $\cdot$ Kidney diseases $\cdot$ Oxidative stress

\section{Abstract \\ Background/Aims: Plasma fluorescent oxidation products (FLOP) constitute a stable and easily measured biomarker of cumulative oxidative stress. However, their association with chronic kidney disease (CKD) has not been studied. Meth- ods: We examined the association of FLOP and CKD in 201 CKD patients and 201 controls without CKD from the com- munity. CKD was defined as an estimated glomerular filtra- tion rate (eGFR) $<60 \mathrm{ml} / \mathrm{min} / 1.73 \mathrm{~m}^{2}$ or the presence of al- buminuria. Results: Adjusted median (interquartile range) FLOP levels were significantly higher in patients with CKD than in controls [FLOP1 (lipid oxidation products): 215.2 (181.3-268.7) vs. 156.6 (139.6-177.3) fluorescent intensity units/ml, p < 0.0001; FLOP2 (DNA oxidation products): 534.8 (379.3-842.4) vs. 269.9 (232.4-410.5) fluorescent intensity units $/ \mathrm{ml}, \mathrm{p}<0.0001$; FLOP3 (protein and phospholipid oxida- tion products): 51.4 (44.4-66.0) vs. 45.2 (38.3-51.7) fluores-}

cent intensity units $/ \mathrm{ml}, \mathrm{p}=0.002]$. Compared with those with a FLOP level below the 75th percentile, participants with a FLOP level above the 75th percentile had increased odds of CKD after adjustment for covariables (FLOP1: odds ratio 13.1, 95\% confidence interval 6.2-27.6; FLOP2: odds ratio 5.7, 95\% confidence interval 2.9-11.1; FLOP3: odds ratio $2.4,95 \%$ confidence interval 1.2-4.7). Levels of FLOP1, FLOP2 and FLOP3 were related to eGFR ( $p<0.0001$ for all) and log-transformed urine albumin ( $p<0.005$ for all) in multivariable-adjusted linear regression models. Conclusion: These data indicate that an elevated FLOP level is associated with CKD status and severity. Future studies are warranted to elucidate its role in the development and progression of CKD.

Copyright $\odot 2012$ S. Karger AG, Basel

\section{Introduction}

Chronic kidney disease (CKD) is a major clinical and public health issue due to its high prevalence, the progression to end-stage renal disease and the disproportionate risk of cardiovascular disease [1]. Traditional risk factors do not sufficiently predict CKD morbidity and mortality.

\section{KARGER}

Fax +4161306 1234

E-Mail karger@karger.ch

www.karger.com
(C) 2012 S. Karger AG, Basel

0250-8095/12/0364-0297\$38.00/0

Accessible online at:

www.karger.com/ajn
Jing Chen, MD, MSc

Department of Medicine, Tulane University School of Medicine

1430 Tulane Ave., SL-45

New Orleans, LA 70112 (USA)

Tel. +1 504988 5346, E-Mail jchen@ tulane.edu 
Recently, it has been suggested that oxidative stress, one of the most potent inducers of endothelial dysfunction and atherosclerosis, is increased in patients with endstage renal disease [2]. However, the association of oxidative stress with predialysis CKD has not been well studied.

Oxidative stress refers to a state of dysmetabolism in chronic disease characterized by an abundance of oxidized macromolecules, including lipids, carbohydrates, proteins, amino acids and DNA, that can result in tissue injury and disease through inflammation and endothelial dysfunction pathways [3,4]. Fluorescent oxidation products (FLOP) result from the interaction of reactive oxygen intermediates and free radicals with macromolecules and show characteristic fluorescence spectra [5]. Commonly used oxidation markers such as $\mathrm{F}_{2}$-isoprostanes and malondialdehyde reflect only a part of oxidation, namely lipid oxidation. FLOP reflect oxidation products generated from several pathways including lipid, protein, DNA and carbohydrate oxidation. The fluorescent method of detecting levels of oxidation products provides a more stable, easily measured and sensitive biomarker of cumulative metabolic and oxidative stress relative to existing techniques for the measurement of oxidative stress biomarkers, such as high-pressure liquid chromatography for measuring malondialdehyde and gas chromatography/mass spectrometry for measuring $\mathrm{F}_{2^{-}}$ isoprostanes [6]. In addition, plasma FLOP independently predicted the risk of subsequent coronary heart disease and other fatal and nonfatal cardiovascular disease events in previous epidemiologic studies [7, 8]. However, the association of FLOP with CKD has not previously been studied. In the present study, we examined the association of FLOP levels and CKD in 201 CKD patients and 201 controls without CKD from the community.

\section{Methods}

\section{Study Participants}

We recruited $201 \mathrm{CKD}$ patients and 201 controls without CKD in the greater New Orleans, La., USA, area from 2007 to 2010. CKD patients aged 21-74 years were recruited from nephrology and internal medicine clinics via physicians' referral by trained research staff in the study area. All eligible CKD patients identified in the recruiting clinics were invited to participate in the study. CKD was defined as an estimated glomerular filtration rate (eGFR) $<60 \mathrm{ml} / \mathrm{min} / 1.73 \mathrm{~m}^{2}$ or the presence of albuminuria $(\geq 30$ $\mathrm{mg} / 24 \mathrm{~h}$ ). Patients were excluded if they had a history of chronic dialysis, kidney transplants, immunotherapy in the past 6 months, chemotherapy within the past 2 years and current clinical trial participation that may have an impact on CKD. Additional exclusion criteria were a history of HIV or AIDS and inability or unwillingness to give informed consent. Controls were recruited through mass mailing to residents aged $21-74$ years living in the same area according to zip code. The eligibility of controls was assessed by a prescreening telephone interview and a clinic screening visit. Individuals were included if they had no evidence of CKD (eGFR $>60 \mathrm{ml} / \mathrm{min} / 1.73 \mathrm{~m}^{2}$ and no persistent albuminuria). Cases and controls were frequency-matched according to age group (10 years), gender and race to increase the efficiency of patient recruitment and statistical analysis.

The Tulane University Institutional Review Board approved this study. Written documentation of informed consent was obtained at the screening visit from all study participants. The procedures followed were in accordance with the ethical standards of the Tulane University Institutional Review Board and the Helsinki Declaration.

\section{Measurements}

A standard questionnaire was administered by trained staff at a clinical visit to obtain demographic information (age, gender, race, education) and information on lifestyle risk factors (including cigarette smoking, alcohol drinking and physical activity), self-reported history of cardiovascular disease, diabetes, hypercholesterolemia and hypertension, as well as the use of antihypertensive, lipid-lowering and diabetes medications and the use of aspirin.

Three blood pressure measurements were obtained at a clinical visit by trained and certified staff according to a common protocol adapted from procedures recommended by the American Heart Association. A standard mercury sphygmomanometer was used, and one of four cuff sizes (pediatric, regular adult, large or thigh) was chosen on the basis of the circumference of the participant's arm. Body height and weight were obtained by trained staff and used to calculate body mass index (body mass index = weight in kilograms/height in meters squared).

An overnight fasting blood sample was collected to measure plasma glucose, serum cholesterol, serum creatinine (SCr) and plasma FLOP. Plasma samples were centrifuged at 3,000 rpm for 45 min within 5 min of sample collection. Serum samples were centrifuged at 2,400 rpm for $20 \mathrm{~min}$ within $10 \mathrm{~min}$ of sample collection. Renal function parameters and a lipid profile were run within $24 \mathrm{~h}$ on a serum sample for each participant. Remaining serum and plasma tubes were frozen at $-80^{\circ} \mathrm{C}$ immediately after centrifugation until further laboratory analysis. A 24-hour urine sample was collected to measure creatinine and albumin.

The procedures for measuring FLOP were adapted from the method of Shimasaki and have been described in detail previously $[6,9]$. Briefly, plasma samples were mixed with ethanol/ ether and centrifuged for $10 \mathrm{~min}$ at 3,000 rpm, after which supernatant was added to cuvettes for spectrofluorometric reading. Fluorescence was quantified as relative fluorescent intensity units per milliliter of plasma at wavelengths of 360/420 nm (excitation/ emission) for FLOP1, 320/420 nm for FLOP2 and 400/475 nm for FLOP3 by a spectrofluorometer (Shimadzu RF-5301 PC). FLOP1 reflects lipid oxidation products that have reacted with proteins, DNA and carbohydrates; FLOP2 can result from the oxidation of linoleate with DNA in the presence of metals, and FLOP3 can be produced by malondialdehyde reacting with proteins and phospholipids [5].

Plasma glucose was measured using a hexokinase enzymatic method (Roche Diagnostics, Indianapolis, Ind., USA). Serum total cholesterol and triglycerides were assayed using an enzymatic procedure on an Hitachi 902 automatic analyzer (Roche Diagnostics). Serum high-density lipoprotein was quantified by a combined 
Table 1. Characteristics of 201 patients with CKD and 201 controls

\begin{tabular}{llcc}
\hline Variable & $\begin{array}{l}\text { CKD patients } \\
(\mathrm{n}=201)\end{array}$ & $\begin{array}{l}\text { Non-CKD controls } \\
(\mathrm{n}=201)\end{array}$ & $\begin{array}{c}\text { p value for } \\
\text { difference }\end{array}$ \\
\hline Mean age \pm SD, years & $55.9 \pm 9.9$ & $52.5 \pm 10.0$ & 0.0007 \\
Male, \% & 55.2 & 45.3 & 0.046 \\
Black, \% & 60.7 & 51.2 & 0.056 \\
High school education, \% & 58.5 & 81.6 & $<0.0001$ \\
Current cigarette smoking, \% & 53.7 & 48.8 & 0.32 \\
Weekly alcohol drinking, \% & 27.9 & 59.2 & $<0.0001$ \\
Twice weekly physical activity, \% & 53.0 & 72.9 & $<0.0001$ \\
Body mass index, kg/m ${ }^{2}$ & $32.2 \pm 7.8$ & $28.9 \pm 6.4$ & $<0.0001$ \\
Mean systolic blood pressure \pm SD, mm Hg & $132.2 \pm 21.0$ & $122.0 \pm 14.7$ & $<0.0001$ \\
Mean diastolic blood pressure \pm SD, mm Hg & $77.2 \pm 13.5$ & $77.6 \pm 9.4$ & 0.77 \\
Mean plasma glucose \pm SD, mg/dl & $119.9 \pm 46.7$ & $103.4 \pm 35.4$ & $<0.0001$ \\
Mean LDL cholesterol \pm SD, mg/dl & $103.3 \pm 45.9$ & $118.2 \pm 30.2$ & $<0.0001$ \\
History of cardiovascular disease, \% & 43.7 & 7.0 & $<0.0001$ \\
History of hypertension, \% & 88.1 & 23.9 & $<0.0001$ \\
History of diabetes, \% & 49.3 & 5.5 & $<0.0001$ \\
History of hypercholesterolemia, \% & 65.7 & 30.8 & $<0.0001$ \\
Antihypertensive medication use, \% & 79.6 & 15.4 & $<0.0001$ \\
Diabetes medication use, \% & 34.3 & 3.0 & $<0.0001$ \\
Lipid-lowering medication use, \% & 21.9 & 9.0 & 0.003 \\
Aspirin use, \% & 34.8 & 8.5 & $<0.0001$ \\
Mean SCr \pm SD, mg/dl & $2.0 \pm 1.1$ & $0.9 \pm 0.2$ & $<0.0001$ \\
Mean eGFR \pm SD, ml/min/1.73 m ${ }^{2}$ & $43.3 \pm 19.9$ & $96.7 \pm 16.8$ & $<0.0001$ \\
Median urine albumin (interquartile range), mg/24h & $74.5(12.3,417.4)$ & $5.9(4.1,11.4)$ & $<0.0001$ \\
\hline
\end{tabular}

SD = Standard deviation; $\mathrm{LDL}=$ low-density lipoprotein; SCr = serum creatinine; eGFR = estimated glomerular filtration rate; $\mathrm{CKD}=$ chronic kidney disease.

procedure of heparin-calcium precipitation of apolipoprotein Bcontaining lipoproteins and agar-agarose gel electrophoresis of lipoproteins. Low-density lipoprotein (LDL) cholesterol was calculated using the Friedewald equation for individuals with serum triglyceride $<400 \mathrm{mg} / \mathrm{dl}$. SCr was measured using the Roche enzymatic method (Roche-Hitachi P-Module instrument with Roche Creatininase Plus assay, Hoffman-La Roche, Basel, Switzerland). Urine concentrations of albumin and creatinine were measured with a DCA 2000 Analyzer (Bayer AG, Leverkusen, Germany). eGFR was estimated from SCr, sex, age and race using the Chronic Kidney Disease Epidemiology Collaboration equation: eGFR = $141 \times \min (\mathrm{SCr} / \kappa, 1)^{\alpha} \times \max (\mathrm{SCr} / \kappa, 1)^{-1.209} \times 0.993^{\text {age }} \times 1.018$ (if female) $\times 1.159$ (if black), where $\kappa$ is 0.7 and 0.9 and $\alpha$ is -0.329 and -0.411 for females and males, respectively [10].

\section{Statistical Analysis}

Basic descriptive statistics were run to describe the demographic and clinical characteristics of the study population. In addition, $t$ tests and $\chi^{2}$ tests were performed to assess the statistical significance of differences in demographic and clinical characteristics according to CKD case or control status.

Inverse transformations were used for plasma FLOP and log transformations were used for urine albumin levels because they were not normally distributed. Multivariable linear regression was used to examine the association between eGFR and inverse- transformed plasma FLOP levels, as well as for log-transformed albumin and inverse-transformed plasma FLOP levels. Age, gender, race, high school education, current cigarette smoking, weekly alcohol consumption, physical activity ( $\geq$ twice per week), body mass index, LDL cholesterol, plasma glucose, systolic blood pressure, history of hypertension, diabetes and cardiovascular disease and medication use were selected for adjustment in these analyses based on their association with CKD in this study and previously published literature. Pearson correlation coefficients were also calculated to assess the association between eGFR and inverse-transformed plasma FLOP levels, as well as the association between log-transformed albumin and inverse-transformed plasma FLOP levels. Quantile regression was used to obtain medians and interquartile ranges of plasma FLOP for CKD patients and controls, after adjusting for the aforementioned covariates [11]. The Wald test was used to test differences in the adjusted medians between CKD patients and controls. Multivariable logistic regression was used to obtain adjusted odds ratios comparing the highest quartile of plasma FLOP levels to the lower three quartiles between CKD patients and controls, after adjustment for the aforementioned covariates. Plasma FLOP quartiles were defined based upon plasma FLOP measurements in the control group. All analyses were performed using SAS version 9.2 statistical software (SAS, Cary, N.C., USA). 
Table 2. Multivariable-adjusted regression coefficients of 1 standard deviation higher in inverse-transformed plasma FLOP with eGFR and log-transformed urine albumin

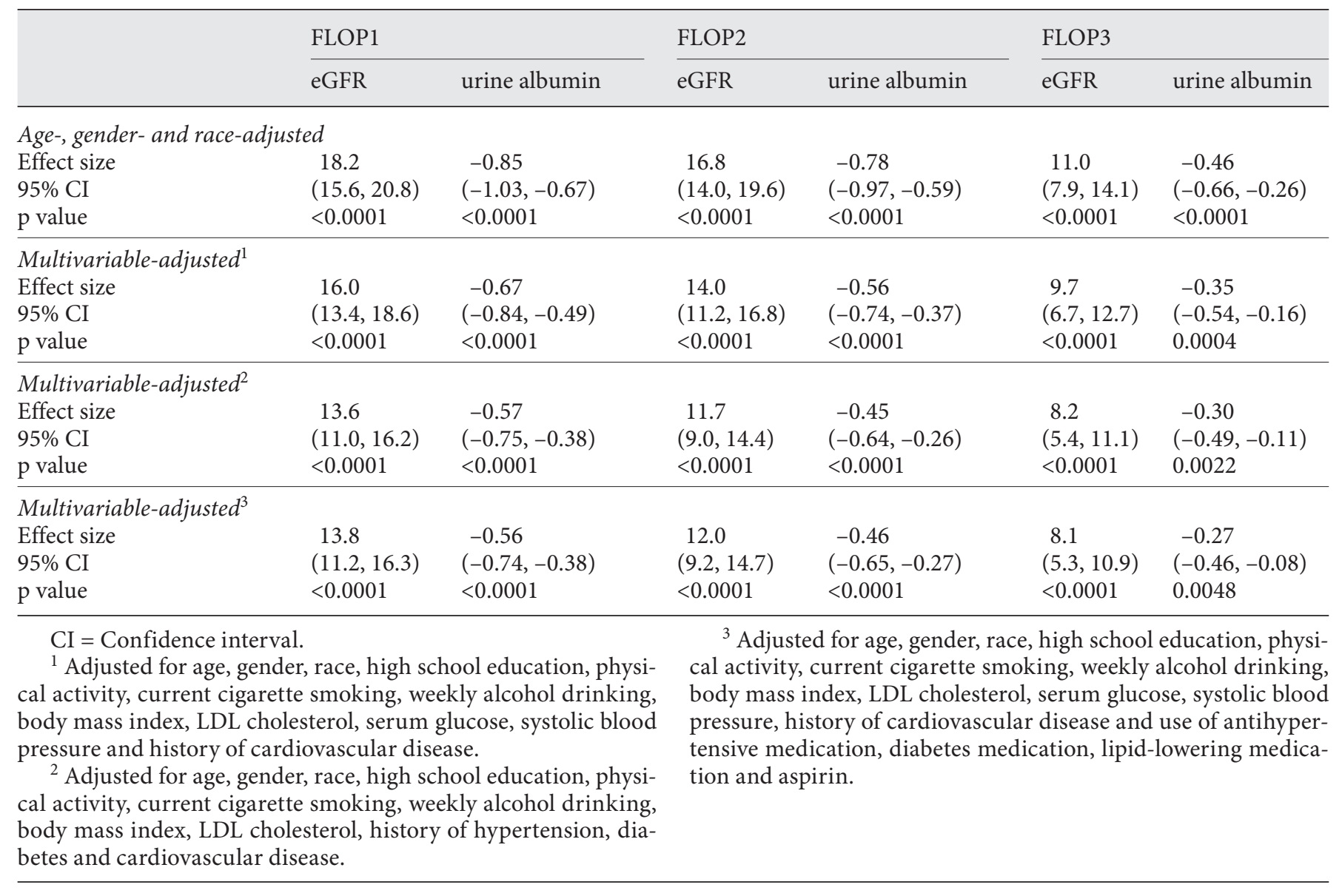

\section{Results}

The demographic and clinical characteristics of the study participants according to CKD status are provided in table 1. Participants with CKD were older, more likely to be male, achieved a lower level of education, were less physically active and less likely to drink alcohol compared to those without CKD. Those with CKD were more likely to have a medical history of cardiovascular disease, hypertension, diabetes and hypercholesterolemia and to be taking medications for these conditions. Mean body mass index, systolic blood pressure, fasting glucose, urine albumin and creatinine were significantly higher while LDL cholesterol and eGFR were lower in CKD patients compared to controls.

The levels of plasma FLOP1, FLOP2 and FLOP3 were significantly related to CKD severity, as indicated by the level of eGFR and urine albumin (table 2; fig. 1a-f). In the multivariable linear regression analyses, inverse-transformed FLOP predicted eGFR ( $p<0.0001$ for all) and log-transformed urine albumin ( $\mathrm{p}<0.005$ for all). Inverse-transformed plasma levels of FLOP1, FLOP2 and FLOP3 were significantly correlated with eGFR $(r=0.58$, 0.55 and 0.38 , respectively; $\mathrm{p}<0.0001$ for all) and logtransformed urine albumin $(\mathrm{r}=-0.45,-0.40$ and -0.25 , respectively; $\mathrm{p}<0.0001$ for all).

Plasma levels of FLOP1, FLOP2 and FLOP3 were consistently higher among CKD patients compared to controls (table 3). After adjustment for age, gender, race, high school education, physical activity, current cigarette smoking, weekly alcohol drinking, body mass index, LDL cholesterol, glucose, systolic blood pressure, history of cardiovascular disease and use of antihypertensive medication, diabetes medication, lipid-lowering agents and aspirin, the median plasma FLOP levels were statistically significantly higher in CKD patients compared to 

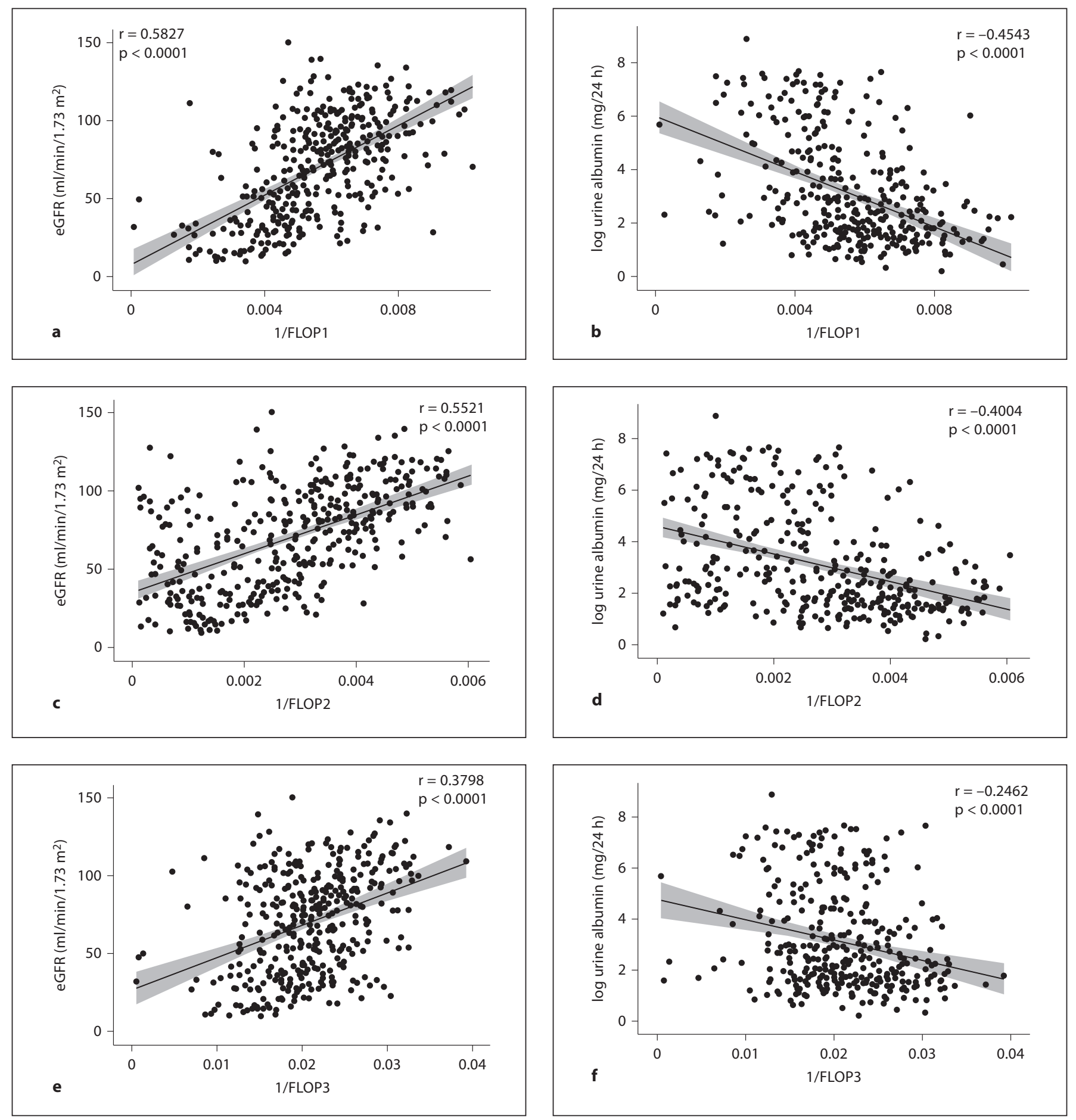

Fig. 1. a-f Scatterplots of inverse-transformed levels of plasma FLOP1, FLOP2 and FLOP3 and eGFR (a, c, e) and log-transformed 24-hour urine albumin levels $(\mathbf{b}, \mathbf{d}, \mathbf{f})$ with fitted regression lines. The shaded area represents the $95 \%$ confidence interval. 
Table 3. Multivariable-adjusted medians of plasma FLOP according to case or control status

\begin{tabular}{|c|c|c|c|c|c|c|c|c|c|}
\hline & \multicolumn{3}{|l|}{ FLOP1, FI/ml } & \multicolumn{3}{|l|}{ FLOP2, FI/ml } & \multicolumn{3}{|c|}{ FLOP3, FI/ml } \\
\hline & cases & controls & $\mathrm{p}$ value & cases & controls & $\mathrm{p}$ value & cases & controls & $\mathrm{p}$ value \\
\hline $\begin{array}{l}\text { Age-, gender- and } \\
\text { race-adjusted }\end{array}$ & $\begin{array}{l}214.7 \\
(180.1,266.2)\end{array}$ & $\begin{array}{l}157.4 \\
(137.6,178.4)\end{array}$ & $<0.0001$ & $\begin{array}{l}511.3 \\
(355.9,909.5)\end{array}$ & $\begin{array}{l}268.6 \\
(231.2,342.5)\end{array}$ & $<0.0001$ & $\begin{array}{l}52.5 \\
(43.7,64.8)\end{array}$ & $\begin{array}{l}44.3 \\
(38.3,51.1)\end{array}$ & $<0.0001$ \\
\hline Multivariable-adjusted $^{1}$ & $\begin{array}{l}216.9 \\
(182.3,272.4)\end{array}$ & $\begin{array}{l}157.8 \\
(139.7,177.5)\end{array}$ & $<0.0001$ & $\begin{array}{l}510.7 \\
(358.2,875.6)\end{array}$ & $\begin{array}{l}270.3 \\
(230.4,376.9)\end{array}$ & $<0.0001$ & $\begin{array}{l}52.5 \\
(44.7,66.3)\end{array}$ & $\begin{array}{l}45.2 \\
(38.2,52.0)\end{array}$ & 0.0001 \\
\hline Multivariable-adjusted $^{2}$ & $\begin{array}{l}216.4 \\
(182.3,267.4)\end{array}$ & $\begin{array}{l}158.2 \\
(139.3,177.4)\end{array}$ & $<0.0001$ & $\begin{array}{l}506.1 \\
(352.7,874.0)\end{array}$ & $\begin{array}{l}265.4 \\
(230.5,384.2)\end{array}$ & $<0.0001$ & $\begin{array}{l}52.0 \\
(44.8,65.1)\end{array}$ & $\begin{array}{l}44.6 \\
(38.4,51.6)\end{array}$ & 0.0008 \\
\hline Multivariable-adjusted ${ }^{3}$ & $\begin{array}{l}215.2 \\
(181.3,268.7)\end{array}$ & $\begin{array}{l}156.6 \\
(139.6,177.3)\end{array}$ & $<0.0001$ & $\begin{array}{l}534.8 \\
(379.3,842.4)\end{array}$ & $\begin{array}{l}269.9 \\
(232.4,410.5)\end{array}$ & $<0.0001$ & $\begin{array}{l}51.4 \\
(44.4,66.0)\end{array}$ & $\begin{array}{l}45.2 \\
(38.3,51.7)\end{array}$ & 0.0019 \\
\hline
\end{tabular}

Figures in parentheses represent interquartile ranges. FI = Fluorescent intensity units.

${ }^{1}$ Adjusted for age, gender, race, high school education, physical activity, current cigarette smoking, weekly alcohol drinking, body mass index, LDL cholesterol, plasma glucose, systolic blood pressure and history of cardiovascular disease.

${ }^{2}$ Adjusted for age, gender, race, high school education, physical activity, current cigarette smoking, weekly alcohol drinking,

body mass index, LDL cholesterol, history of hypertension, history of diabetes and history of cardiovascular disease.

${ }^{3}$ Adjusted for age, gender, race, high school education, physical activity, current cigarette smoking, weekly alcohol drinking, body mass index, LDL cholesterol, plasma glucose, systolic blood pressure, history of cardiovascular disease and use of antihypertensive medication, diabetes medication, lipid-lowering medication and aspirin.

Table 4. Odds ratios of CKD associated with the top quartile compared with the three lower quartiles of FLOP1, 2 and 3

\begin{tabular}{|c|c|c|c|c|c|c|}
\hline & \multicolumn{2}{|l|}{ FLOP1 } & \multicolumn{2}{|l|}{ FLOP2 } & \multicolumn{2}{|l|}{ FLOP3 } \\
\hline & OR & $\mathrm{p}$ value & OR & $\mathrm{p}$ value & OR & $\mathrm{p}$ value \\
\hline Age-, gender- and race-adjusted & $10.0(6.2,16.1)$ & $<0.0001$ & $8.3(5.1,13.2)$ & $<0.0001$ & $2.5(1.6,4.0)$ & $<0.0001$ \\
\hline Multivariable-adjusted ${ }^{1}$ & $10.2(5.6,18.8)$ & $<0.0001$ & $6.7(3.8,11.8)$ & $<0.0001$ & $2.6(1.5,4.6)$ & 0.001 \\
\hline Multivariable-adjusted $^{2}$ & $10.4(5.1,21.4)$ & $<0.0001$ & $4.7(2.5,8.9)$ & $<0.0001$ & $3.1(1.6,6.2)$ & 0.001 \\
\hline Multivariable-adjusted ${ }^{3}$ & $13.1(6.2,27.6)$ & $<0.0001$ & $5.7(2.9,11.1)$ & $<0.0001$ & $2.4(1.2,4.7)$ & 0.0109 \\
\hline
\end{tabular}

Figures in parentheses represent $95 \%$ confidence intervals. $\mathrm{OR}=$ Odds ratio.

${ }^{1}$ Adjusted for age, gender, race, high school education, physical activity, current cigarette smoking, weekly alcohol drinking, body mass index, LDL cholesterol, plasma glucose, systolic blood pressure and history of cardiovascular disease.

${ }^{2}$ Adjusted for age, gender, race, high school education, physical activity, current cigarette smoking, weekly alcohol drinking, body mass index, LDL cholesterol, history of hypertension, history of diabetes and history of cardiovascular disease.

${ }^{3}$ Adjusted for age, gender, race, high school education, physical activity, current cigarette smoking, weekly alcohol drinking, body mass index, LDL cholesterol, plasma glucose, systolic blood pressure, history of cardiovascular disease and use of antihypertensive medication, diabetes medication, lipid-lowering agents and aspirin. controls for FLOP1, FLOP2 and FLOP3 ( $\mathrm{p}<0.002$ for all).

Logistic regression analyses demonstrated a strong, significant and consistent relationship between plasma levels of FLOP1, FLOP2 and FLOP3 and CKD status (table 4). For example, participants in the top quartile ( $\geq 75$ th percentile) of plasma FLOP1 had a 13 -fold increased odds of having CKD relative to those partici- pants with lower levels of plasma FLOP1, after adjusting for age, gender, race, education, physical activity, cigarette smoking, alcohol drinking, body mass index, LDL cholesterol, glucose, systolic blood pressure, history of cardiovascular disease and use of antihypertensive medication, diabetes medication, lipid-lowering agents and aspirin. 


\section{Discussion}

These study findings indicate that plasma levels of FLOP1, FLOP2 and FLOP3 were significantly higher in CKD patients compared to controls without CKD, even after adjustment for traditional risk factors for CKD and other covariates including demographic and behavioral characteristics, clinical and anthropometric measurements, history of related diseases and medication use. Furthermore, we document a strong dose-response relationship between plasma levels of FLOP1, FLOP2 and FLOP3 and the degree of kidney impairment using both eGFR and urine albumin as markers.

A few clinical studies have demonstrated an increase in oxidative stress among CKD patients using a myriad of other biomarkers, including malondialdehyde, oxidized lipids, $\mathrm{F}_{2}$-isoprostanes, asymmetric dimethylarginine, advanced glycation end products and thiols [12-14]. For instance, one small study detected a graded relationship between CKD status and advanced glycosylation end products among 8 subjects with normal renal function (16.8 units/ml), 6 subjects with elevated SCr and persistent proteinuria (30.2 units $/ \mathrm{ml}$ ) and 6 subjects with end-stage renal disease (82.8 units $/ \mathrm{ml}$ ) [12]. In a case-control study of 60 cases with stage 3-5 CKD and 70 healthy controls, levels of carbonyls and $\mathrm{F}_{2}$-isoprostanes were statistically significantly higher among cases than controls [13]. However, these previous oxidative stress studies were mainly conducted in patients with severe kidney disease and renal failure. The present study includes patients with moderate kidney disease and demonstrates the positive association between FLOP and the severity of CKD. As such, oxidative stress may play a role in the etiopathogenesis of CKD. In addition, FLOP has been shown to be stable in samples and reproducible within subjects over time $[6,7$, 15]. Furthermore, the measurement of FLOP can be performed on samples that are collected with standard procedures, and the laboratory technique is relatively uncomplicated whereas other oxidative stress markers require specific sample preparation and more sophisticated laboratory techniques such as liquid chromatography and mass spectrometry $[6,15]$. These features of FLOP suggest it may be a useful marker for CKD risk stratification in clinical settings and for research studies.

The underlying etiology for increased oxidative stress is not fully understood. The proximal tubular cells in the kidney have a direct role in creating damaging oxidative products through the production of hydrogen peroxide in myoglobinuria [16]. A major component of the kidney's role in determining the degree of systemic oxidative stress is through the retention of oxidative products due to the loss of kidney function [17]. Damaged kidneys have decreased antioxidant activity and thereby accumulate oxidative products [18]. Reactive oxygen species have been shown to impact the absorption and excretion of sodium chloride and water in the thick ascending limb of the loop of Henle and the medulla [19]. Deficiency of superoxide dismutase, which metabolizes reactive oxygen species, leads to endothelial dysfunction, enhances afferent arteriole sensitivity to angiotensin II and promotes remodeling of the renal vasculature [20]. Reactive oxygen species have been shown to directly mediate renal vasoconstriction and blood flow through the kidney $[19,21]$. Our study suggests that there is markedly increased oxidative stress in CKD patients compared to controls. Elevated oxidative stress likely induces kidney dysfunction through the endothelial dysfunction and inflammation pathways.

Even though randomized clinical trials of antioxidant vitamins have not demonstrated a beneficial effect on cardiovascular disease in women [22], $\mathrm{N}$-acetylcysteine and $\alpha$-tocopherol (vitamin E) have both been shown to reduce cardiovascular disease events in end-stage renal disease patients in randomized clinical trials $[23,24]$. Recently, bardoxolone methyl, a newly developed antioxidant inflammation modulator, showed some evidence of improving eGFR among diabetic CKD patients in a clinical trial [25]. These studies corroborate the theory that oxidative stress is involved with CKD pathology and that targeting oxidative stress pathways could delay or reverse $\mathrm{CKD}$ progression. As new antioxidant medications continue to become available, the plasma FLOP level could be tested as a marker to guide treatment and monitor therapeutic effects. Furthermore, clinical interventions which decrease FLOP levels may improve renal function.

There are several noteworthy strengths of this study. Our study is the first to find that plasma FLOP was significantly increased in patients with CKD. In addition, we discovered a dose-response relationship between the plasma level of FLOP and the severity of CKD as measured by both eGFR and albuminuria. This association was independent of multiple CKD covariables that were carefully collected during the study. Furthermore, given the diverse study population, these results are generalizable for both men and women and both African-Americans and Caucasians.

There are a few potential limitations of this study. Due to the observational nature of this study, there is the potential for residual confounding. However, we carefully collected and adjusted for multiple lifestyle factors, lipid 
markers and other known risk factors for CKD. Secondly, the cross-sectional design limits the ability to infer causality between the FLOP level and CKD. Future studies that prospectively identify CKD outcomes associated with the baseline plasma level of FLOP are warranted. Lastly, FLOP is a nonspecific biomarker of oxidative stress and reflects oxidation of proteins, lipids and DNA. However, FLOP provides a better indication of cumulative oxidative damage than those markers that measure a single macromolecule.

In summary, this study indicates that oxidative stress is associated with CKD status and severity. Future studies are warranted to elucidate its role in the development and progression of CKD.

\section{Acknowledgements}

This study was supported by a grant (P20-RR017659) from the National Center for Research Resources, National Institutes of Health, Bethesda, Md., USA, and partially supported by a Clinical and Translational Clinical Research Center grant from the Louisiana Board of Regents Research Commercialization and Educational Enhancement Program Fund.

\section{Disclosure Statement}

The authors declare no conflict of interest.

\section{References}

1 Hemmelgarn BR, Manns BJ, Lloyd A, James MT, Klarenbach S, Quinn RR, Wiebe N, Tonelli M; Alberta Kidney Disease Network: Relation between kidney function, proteinuria, and adverse outcomes. JAMA 2010;303: 423-429.

2 Schnabel R, Blankenberg S: Oxidative stress in cardiovascular disease: successful translation from bench to bedside? Circulation 2007;116:1338-1340.

-3 Papa S, Skulachev VP: Reactive oxygen species, mitochondria, apoptosis and aging. Mol Cell Biochem 1997;174:305-319.

4 Marnett LJ, Riggins JN, West JD: Endogenous generation of reactive oxidants and electrophiles and their reactions with DNA and protein. J Clin Invest 2003;111:583-593.

5 Frankel EN: Lipid Oxidation. Dundee, The Oily Press, 1998.

-6 Wu T, Rifai N, Roberts LJ, Willett WC, Rimm EB: Stability of measurements of biomarkers of oxidative stress in blood over 36 h. Cancer Epidemiol Biomarkers Prev 2004; 13:1399-1402.

7 Wu T, Rifai N, Willett WC, Rimm EB: Plasma fluorescent oxidation products: independent predictors of coronary heart disease in men. Am J Epidemiol 2007;166:544-551

8 Mezzetti A, Zuliani G, Romano F, Costantini F, Pierdomenico SD, Cuccurullo F, Fellin R; Associazione Medica Sabin: Vitamin E and lipid peroxide plasma levels predict the risk of cardiovascular events in a group of healthy very old people. J Am Geriatr Soc 2001;49: 533-537.

-9 Shimasaki H: Assay of fluorescent lipid peroxidation products. Methods Enzymol 1994; 233:338-346.

10 Levey AS, Coresh J, Greene T, Stevens LA, Zhang YL, Hendriksen S, Kusek JW, Van Lente F; Chronic Kidney Disease Epidemiology Collaboration: Using standardized serum creatinine values in the modification of diet in renal disease study equation for estimating glomerular filtration rate. Ann Intern Med 2006;145:247-254.

11 McGreevy KM, Lipsitz SR, Linder JA, Rimm E, Hoel DG: Using median regression to obtain adjusted estimates of central tendency for skewed laboratory and epidemiologic data. Clin Chem 2009;55:165-169.

-12 Makita Z, Radoff S, Rayfield EJ, Yang Z, Skolnik E, Delaney V, Friedman EA, Cerami A, Vlassara H: Advanced glycosylation end products in patients with diabetic nephropathy. N Engl J Med 1991;325:836-842.

13 Oberg BP, McMenamin E, Lucas FL, McMonagle E, Morrow J, Ikizler TA, Himmelfarb J: Increased prevalence of oxidant stress and inflammation in patients with moderate to severe chronic kidney disease. Kidney Int 2004;65:1009-1016.

14 Simmons EM, Langone A, Sezer MT, Vella JP, Recupero P, Morrow JD, Himmelfarb J: Effect of renal transplantation on biomarkers of inflammation and oxidative stress in end-stage renal disease patients. Transplantation 2005;79:914-919.

15 Okamura DM, Himmelfarb J: Tipping the redox balance of oxidative stress in fibrogenic pathways in chronic kidney disease. Pediatr Nephrol 2009;24:2309-2319.

16 Zager RA, Burkhart K: Myoglobin toxicity in proximal human kidney cells: roles of $\mathrm{Fe}$, $\mathrm{Ca}^{2+}, \mathrm{H}_{2} \mathrm{O}_{2}$, and terminal mitochondrial electron transport. Kidney Int 1997;51:728738.

17 Himmelfarb J: Relevance of oxidative pathways in the pathophysiology of chronic kidney disease. Cardiol Clin 2005;23:319_ 330.

8 Cvetkovic T, Vlahovic P, Pavlovic D, Kocic G, Jevtovic T, Djordjevic VB: Low catalase activity in rats with ureteral ligation: relation to lipid peroxidation. Exp Nephrol 1998;6: 74-77.
19 Zou AP, Li N, Cowley AW: Production and actions of superoxide in the renal medulla. Hypertension 2001;37:547-553.

20 Carlstrom M, Lai EY, Ma Z, Steege A, Patzak A, Eriksson UJ, Lundberg JO, Wilcox CS, Persson EG: Superoxide dismutase 1 limits renal microvascular remodeling and attenuates arteriole and blood pressure responses to angiotensin II via modulation of nitric oxide bioavailability. Hypertension 2010;56:907-913.

21 Just A, Olson AJ, Whitten CL, Arendshorst WJ: Superoxide mediates acute renal vasoconstriction produced by angiotensin II and catecholamines by a mechanism independent of nitric oxide. Am J Physiol Heart Circ Physiol 2007;292:H83-H92.

22 Cook NR, Albert CM, Gaziano JM, Zaharris E, MacFadyen J, Danielson E, Buring JE, Manson JE: A randomized factorial trial of vitamins $\mathrm{C}$ and $\mathrm{E}$ and beta carotene in the secondary prevention of cardiovascular events in women: results from the women's antioxidant cardiovascular study. Arch Intern Med 2007;167:1610-1618

23 Boaz M, Smetana S, Weinstein T, Matas Z, Gafter U, Iaina A, Knecht A, Weissgarten Y, Brunner D, Fainaru M, Green MS: Secondary prevention with antioxidants of cardiovascular disease in endstage renal disease (SPACE): randomised placebo-controlled trial. Lancet 2000;356:1213-1218.

24 Tepel M, van der Giet M, Statz M, Jankowski J, Zidek W: The antioxidant acetylcysteine reduces cardiovascular events in patients with end-stage renal failure: a randomized, controlled trial. Circulation 2003;107:992-995.

25 Pergola PE, Raskin P, Toto RD, Meyer CJ, Huff JW, Grossman EB, Krauth M, Ruiz S, Audhya P, Christ-Schmidt H, Wittes J, Warnock DG; BEAM Study Investigators: Bardoxolone methyl and kidney function in CKD with type 2 diabetes. N Engl J Med 2011;365:327-336. 